between the velocity and the light intensity (or the amount of absorbed light) in a photochemical change is not constant but changes with the acceleration of the reaction by light absorption ${ }^{4}$.

N. R. Dhar.

A. K. Bhattacharya.

B. L. MukerJi.

Chemical Laboratory,

University of Allahabad, Allahabad.

1 Dhar, Proc. Akad. Wetensch. Amsterdam, 18, $1084 ; 1916$.

2 Berthoud and Bellenot, Helv. Chim. Acta, 7, 307; 1924. Briers Chapman, and Walters, .J. Chem. Soc., 129, 562; 1926. Mukerji an Dhar, J. Phys. Chem., 32, 1308; 1928. Allmand and Young, Farada: Soc. Discuss., 515, April 1931. 'Young and Style, ibid., 493. Griflit! and McKeown, Trans. Faraday Soc., 28, 752; 1932.

${ }^{3}$ Bhattacharya and Dhar, J. Indian Chrm. Soc., 6, 473; 1929

- Dhar and Bhagwat. Z. Anorg. Chem., 190, 415; 1930. Malaviya Dhar and Bhagwat, ibid., 199, 406; 1931. Dhar, "Chemical Action of Light", Blackie and Son, 1931

\section{Ultra-Violet Bands of Oxide of Phosphorus}

THE Phosphorus arc in air gives out a band spectrum in the ultra-violet, which is attributed to the $\mathrm{PO}$ molecule. The vibrational quantum analysis of these bands has been previously done by one of the authors $^{1}$. The rotational structure analysis of the $(0,0)$ band at $\lambda 2477 \cdot 80$ shows that it consists of six main branches, namely, $P_{1} Q_{1} R_{1}$ and $P_{2} Q_{2} R_{2}$ and two other faint satellite branches. For low quantum numbers, the satellite branches $R_{Q_{21}}, Q P_{21}, P_{Q_{12}}$ and $Q_{R_{12}}$ are superposed on the main branches. The intensity of the lines of the different branches satisfy the criterion of a ${ }^{2} \Sigma \rightarrow^{2} \pi$ transition, The band structure is analogous to the v-bands of $\mathrm{NO}_{1}$ as is anticipated from theoretical considerations. The following molecular constants (cm. ${ }^{-1}$ units) have been obtained.

$v_{e}^{\prime}=1.416 \times 10^{-8}$

$\ddot{B}_{e}^{\prime}=1 \cdot 3060$

$D_{e}^{\prime}=-4 \cdot 60 \times 10^{-6}$

$$
\begin{gathered}
v_{e}^{\prime \prime}=1 \cdot 458 \times 10^{-8} \\
B_{e}^{\prime \prime}\left(\frac{\pi}{2}\right)=1 \cdot 2332 ; B_{e}^{\prime \prime}\left(\frac{3 \pi}{2}\right)=1 \cdot 2256 \\
D_{e}^{\prime \prime}\left(\frac{\pi}{2}\right)=\frac{-4}{D_{e}^{\prime \prime}} \cdot 96 \times 10^{-6} ; \\
\alpha^{\prime}=\alpha^{\prime \prime}=0.0073
\end{gathered}
$$

The detailed results will be published elsewhere.

$$
\text { P. N. Ghosh. }
$$

A. K. Sen Gupta.

Applied Physics Laboratory,

University College of Science, Calcutta.

${ }^{1}$ I. N. Ghosh and G. N. Ball, Z. Physik., 71, 362; 1931.

\section{Conductivity of Mixtures of Gases}

$\mathrm{Ir}$ is well known that the electrical conductivity of certain gases may be greatly increased by the addition of very small quantities of other gases. For example, the electrical conductivity of pure helium is greatly improved by the addition of 0.01 per cent of pure argon. In gases, the electrical conductivity in uniform fields between parallel plates depends upon the ratio $X / p$, where $X$ is the electrical intensity in volts per centimetre, and $p$ is the pressure in millimetres of mercury, and is a maximum for a certain value of $X / p$ depending upon the nature of the gas. The photo-electric currents obtained with a constant force between parallel plates at different distances apart are represented by the ordinates of the curves, Fig. 1, and the potential differences between the plates by the abscissæ. The three curves give the currents in pure helium, in pure argon and in helium containing 0.025 per cent of argon. The values of the ratio $X / p$ were, 50 in pure helium, 200 in pure argon and 15 in the mixture. Under these conditions the rate of increase of the current with the distance between the plates was a maximum.

'Two theories have been advanced to account for the increase in conductivity of the mixture, namely, the action of direct collisions of electrons with the atoms of argon, and the action of metastable atoms of helium which are formed by electron collisions

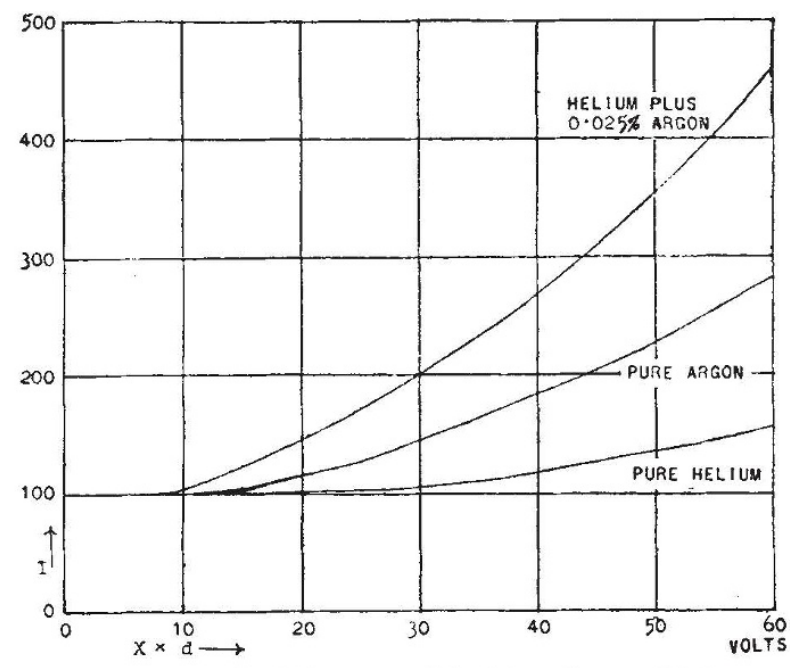

FIG 1-Conductivity between parallel plates. $I$, current between plates in arbitrary units; $X$, forces between plates in volts per centimetre ; $d$, distance between plates in centimetres.

with helium atoms. These metastable atoms of helium may be formed when the electrons attain an energy corresponding to approximately 20 volts. It is then supposed that the metastable atoms of helium ionise the atoms of argon, which require an energy corresponding to approximately 16 volts. There is much controversy concerning the relative importance of these two processes. For example, Penning $^{1}$ states that the increase in conductivity may be attributed entirely to metastable atoms, whereas Townsend and McCallum ${ }^{2}$ have pointed out that the effect of direct collisions of electrons may be considerable in these circumstances.

The results given in Fig. 1 are of importance in showing that a large number of new ions are formed in the mixture of 0.025 per cent of argon in helium when the potential between the plates is increased from 9 volts to 18 volts, the distance between them being adjusted so as to maintain a constant force. As no metastable atoms of helium could be formed until the electrons attained an energy of 20 volts, the increase in conductivity in these circumstances must be attributed to the direct collisions of electrons with argon atoms. It would not appear from the curve that any important new process occurs when the potential between the plates exceeds 20 volts, such as might be attributed to the action of metastable atoms.

More complete results will shortly be published elsewhere.

S. P. McCallum.

Electrical Laboratory,

L. KI Atzow.

Oxford.

April 24.

1 F. M. Penning, Physica, 12, $66 ; 1932$.
J. S. Townsend and S. P. McCallum, Phil. Mag., 5, $695 ; 1928$. 